\title{
AN ENERGY APPROACH TO THE DESIGN OF SINGLE DEGREE OF FREEDOM GRAVITY BALANCERS WITH COMPLIANT JOINTS
}

\author{
Boaz L. Rijff ${ }^{1,2}$ \\ ${ }^{1}$ InteSpring B.V. \\ Delft, The Netherlands \\ Email: boaz@intespring.nl
}

\author{
Just L. Herder ${ }^{2}$ \\ ${ }^{2}$ Delft University of Technology \\ Department of BioMechanical Engineering \\ The Netherlands
}

\section{ABSTRACT}

A gravity balancer is a mechanism that compensates the weight of a mass over a range of motion. When no friction is present, this gives an energy efficient mechanism and little effort is required to move an object. Conventional mechanisms have drawbacks due to the use of conventional rigid joints. Compliant joints do not have these disadvantages, can be made from fewer parts and can increase performance compared to rigid body joints. The goal of this paper is to develop a new method for the design of single degree of freedom gravity balancers where all the rigid joints are replaced with compliant joints. The method is based on connecting rigid links with compliant joints. With a constant potential energy as objective, the method allows new gravity balancers to be designed. The second goal is to construct a demonstrator as proof of principle. It can be concluded that for the first time a gravity balancers has been constructed where all the rigid joints are replaced with compliant joints. The gravity balancer had a peak moment reduction of 93\%. The presented method is extensible and allows others to understand and to further develop gravity balancers with compliant joints for other applications.

Keywords: Static balance, constant potential energy, gravity balancing, compliant joints, torsion stiffness.

\section{INTRODUCTION}

A method to gain energy efficient mechanisms is through static balance. Statically balanced mechanisms are in continuous equilibrium. The system will not just be in equilibrium at certain positions, but will be in equilibrium throughout its entire range of motion. When no friction is present in this system, it implies a constant potential energy [1]. As a result, low actuation forces are required to move the system.
To gain a constant potential energy, the change in potential energy of an object can be balanced by the potential energy of a second energy storage element, e.g. a counterweight or a spring. When a spring is used as energy storage element as opposed to the use of a counterweight, it reduces the total weight and inertia of the system [2].

When a mechanism statically balances the weight of an object it is referred to as a gravity balancer. A gravity balancer allows a weight to be moved up and down with little effort. In the field of industrial robotics for example this allows smaller motors to be used, the size of required brakes can be reduced and inherent safety is introduced [3]. Gravity balancers can also play a large role in height adjustable applications for rehabilitation [4, 5], patient care and in height adjustment of furniture [6].

Numerous gravity balancing solutions have been developed over the years [4]. However, these conventional gravity balancers make use of rigid body joints. The use of these conventional rigid joints causes backlash, friction and wear [7]. These disadvantages can lead to poor accuracy, poor repeatability and can introduce high maintenance costs [8].

To omit the disadvantages of rigid joints, compliant joints can be used instead [8]. Compliant joints gain their motion from the deformation of the material. This results in reduced friction, reduced wear and no need for lubrication. The properties of compliant joints give advantages especially for mechanisms that are hard to reach or have to operate in harsh environments. Further benefits are gained from the monolithic structure of these compliant joints. They allow simplified production and reduced assembly time, which results in low fabrication costs [7]. The rotation of these compliant joints is obtained from the angular displacement of elastic members which implies a torsional stiffness. This stiffness is often considered to be a disadvantage [7,9], as energy is stored in the system as elastic potential. In gravity balancers 
the elastic potential energy of the compliant joints can be used to obtain a constant potential energy. Consequently, the introduced stiffness becomes an advantage.

We propose to develop a gravity balancer where all the rigid joints are replaced with compliant joints. Such a mechanism would combine the advantages of static balancing the weight of a mass with the advantages of compliant joints. Realizing the synthesis of such a mechanism is a difficult task. It is required that every pivot point is modeled with a torsional stiffness. Some examples from literature of gravity balancing with torsional stiffness are discussed next.

Gopalswamy et al. [3] balanced the weight of a parallelogram linkage with a single torsion spring with a linear characteristic. The range of motion was limited to the linear range of the sinusoidal moment curve of the mass. The solution was specific to the application.

Trease and Dede [10] developed a gravity balancer consisting of a four-bar linkage. A constant potential energy was approximated by changing the kinematics of the four-bar linkage. Two of the four joints were replaced with a compliant joint. The applied optimization method was limited to the design of the application.

Claus [11] used a torsion bar to balance the weight of a container wall. He applied a series of physical stops to approximate the desired characteristic, but still relied on rigid joints.

Radaelli et al. [12] developed a general design method to approximate static balance with the use of a balancing segment with torsional stiffness. He balanced a four-bar linkage with a mass connected the end of one of the links. His method did not take the weight of the mechanism into account and was not able to deal with the torsion stiffness at one of the connection points.

None of the mentioned gravity balancers from literature gain their full motion from the deflection of material and still rely on rigid mechanical joints. However their efforts do indicate the possibility for a gravity balancer with compliant joints.

The goal of this paper is to develop a new method for the design of gravity balancers, where all the rigid joints are replaced with compliant joints. The method is based on the requirement of constant potential energy and incorporates a torsion stiffness at every pivot point. The mechanism will consist of linkages connected by compliant joints modeled as torsion springs. The system is assumed to work in a non-horizontal plane. The second goal is to construct a demonstrator. The demonstrator will be measured as proof of principle.

This paper will have the following structure. The second section will discuss the applied design and calculation method. Three examples created with the new design method are presented in Section 3. Section 4 discusses the construction of a demonstrator followed by an evaluation. Section 5 contains the discussion. The conclusions are presented in Section 6.

\section{METHOD}

A method is developed to design a gravity balancer that uses rigid links connected by compliant joints. The method will assume to work with complaint joints with linear stiffness. These compliant joints can be modeled as linear torsion springs [7]. The general term torsion springs or spring will be used to refer to the elastic potential energy of compliant joints.

With $k$ as the torsional stiffness and $\phi_{0}$ as the initial angle, the moment $M$ created as a function of the angular rotation $\phi$ can be written as Eqn. (1). Consequently, the energy stored in the spring $U_{s}$ can be written as in Eqn. (2) [13].

$$
\begin{aligned}
M & =k \cdot\left(\phi-\phi_{0}\right) \\
U_{s} & =\frac{1}{2} \cdot k \cdot\left(\phi-\phi_{0}\right)^{2}
\end{aligned}
$$

Compliant joints can have many different embodiments, but the moment $M$ and energy $U_{s}$ are calculated similarly. Figure 1 shows three examples of compliant joints.

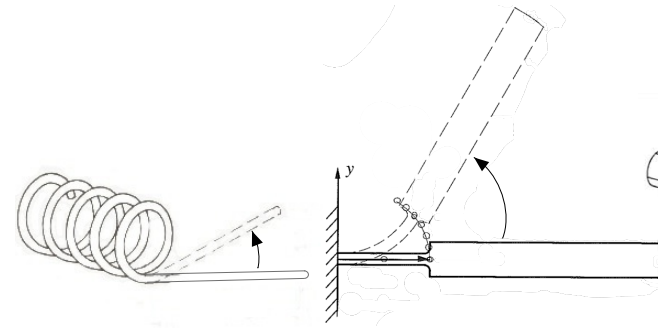

(a) (b)

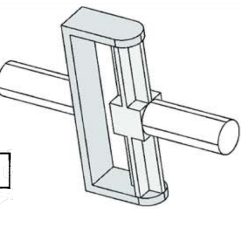

(c)
FIGURE 1: Examples of compliant joints. (a) A helical torsion spring, (b) a small-length flexural pivot (adapted from [7]) and (c) a compliant revolute joint $[8]$

\subsection{DESIGN STEPS}

In the design method, we propose to take various steps before physical construction of a mechanism. The general steps of the design method are summarized in Fig. 22 The method is based on designing a basic balancing component. Any number of balancing components can be connected to an unbalanced function having a single degree of freedom (1-2). The designer is free to chose the type of joints before or after optimizing the parameters. If the weight of the construction is known, this can also be used as input. The parameters that determine the energy curve of the total system can be selected and optimized (3). An optimization algorithm is used to approximate a constant potential energy. 


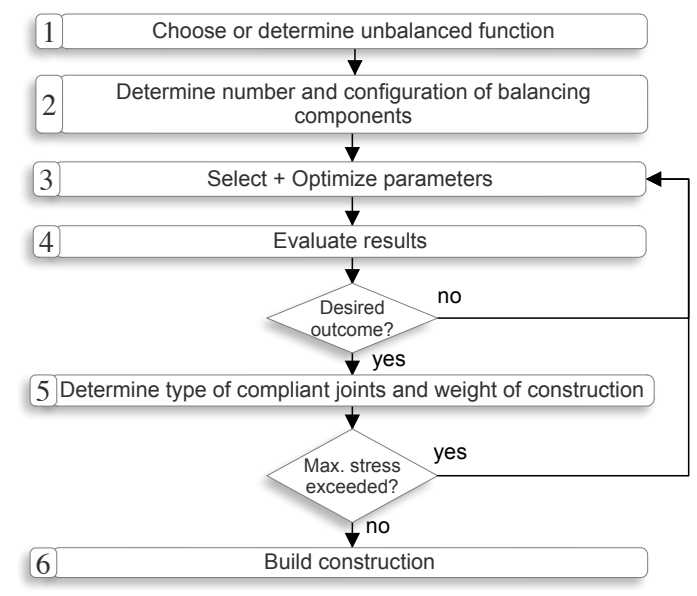

FIGURE 2: A summary of the general steps taken in the design method.

After evaluation of the results (4), the range of the parameters can be adjusted to attempt to find a more desired outcome (4-3). The range of the parameters may also be adjusted so that the maximum stress of a joint is not exceeded (5-3). After a desired outcome has been found, the final step is construction of the mechanism (6). In Fig. 2 the type of joints and weight of the construction are considered to be unknown at the start.

\subsection{Basic balancing component}

The basic balancing component consists of two links connected by torsion springs. The extremities of the links are also connected by torsion springs. Figure 3 shows the geometry of the basic balancing component. The spiral springs represent the torsional stiffness of the compliant joints schematically. The basic balancing component can have two configurations: clockwise and counterclockwise.

This balancing component is similar the one developed by Radaelli et al. [12]. The novelty is the addition of a torsional stiffness at point $C$ and the addition of the gravitational components of the links.

\subsection{Design parameters}

The design parameters of a mechanism are determined by the unbalanced function and number of chosen balancing components. As input the following fixed parameters are selected: the weight of the payload $(m)$, the weight of the construction and the range of motion $(R o M)$.

When the basic elements are know, the design parameters can be selected. These parameters can be optimized to influence the functionality of the mechanism and are considered to be the design vector $\mathbf{v}$ of the mechanism. As example, a mass connected to a rotating link is taken as unbalanced function, Fig. 4 With

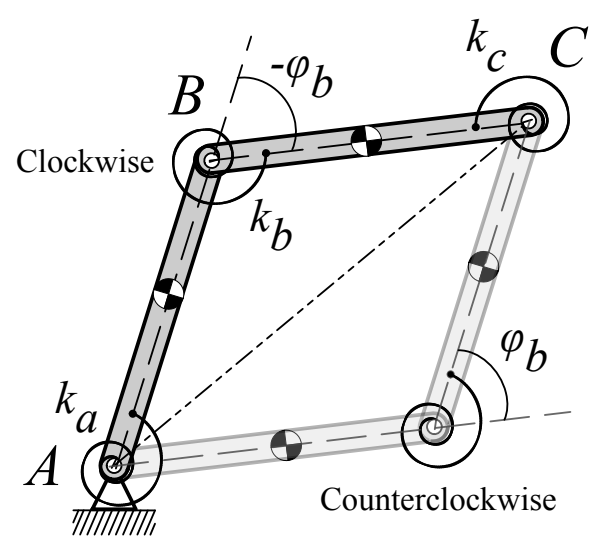

FIGURE 3: The basic balancing component consists of a two-link arm where all the endpoints of the links are compliant joints. The torsional stiffness of the compliant joints are shown schematically as spiral springs. The balancing component can have a clockwise and counterclockwise configuration. In the clockwise configuration angle $\phi_{b}$ is calculated as negative value. Point $A$ is the attachment point of the balancing component to a base. $C$ is the point where the balancing component is connected to the unbalanced function. The two links are connected at point $B$. A torsion stiffness is modeled at every point, where the corresponding stiffnesses are denoted by $k_{a}, k_{b}$ and $k_{c}$.

a single clockwise balancing component connected to the unbalanced function, a four-bar mechanism is created. The design vector of this four-bar mechanism is taken as example. Figure 4 shows the schematic representation of the mathematical model with all the parameters. The payload $m$ and weight of the links are selected as input and do not belong to the design vector. The position of the center of mass of the unbalanced function may differ and is indicated by distance $\rho \cdot r$. The design vector $\mathbf{v}$ can be written as: $\left[x_{0}, y_{0}, \phi_{a 0}, \phi_{b 0}, \phi_{c 0}, \phi_{d 0}, k_{a}, k_{b}, k_{c}, k_{d}, L_{1}, L_{2}, L_{3}, \rho\right]$.

It is possible to select any subset of parameters within the design vector. The remaining parameters are then considered to be fixed. This paper will assume the center of mass to be fixed: $\rho=\frac{1}{2}$.

\subsection{Kinematic relations}

The relationship between the parameters determine the total potential energy curve. Consequently, the position and orientation of the links and torsion springs are required to be known at every position of the payload. Figure 4 shows the angles that need to be known for the kinematic relations.

As degree of freedom $\theta$ is used. $\theta$ is the angle between the $\mathrm{x}$-axis and the rotating link (D-E) connected to the payload. 


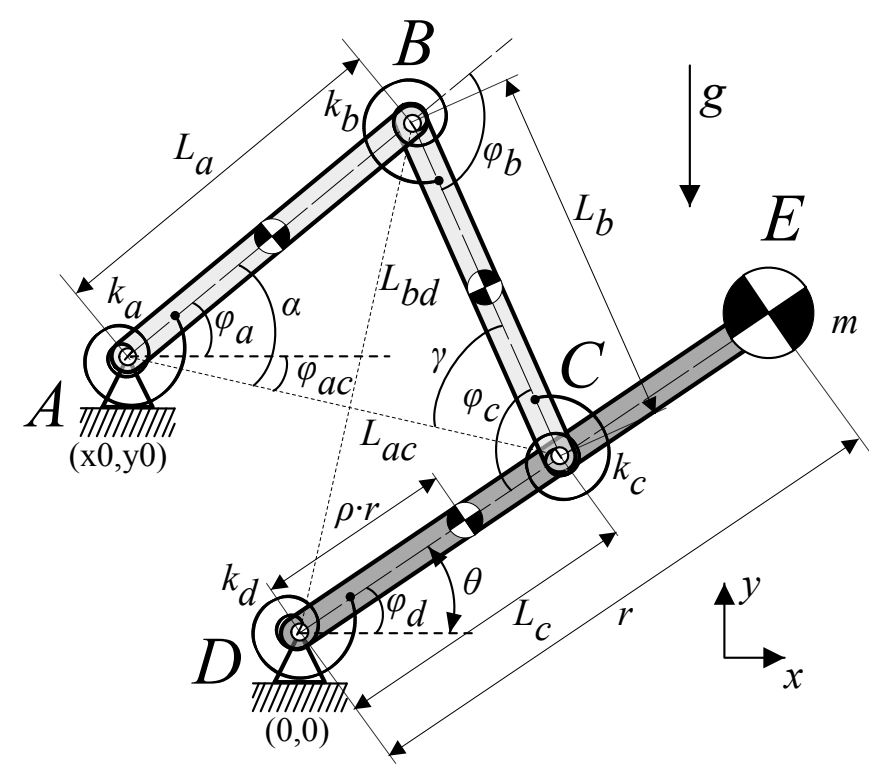

FIGURE 4: The basic balancing component with clockwise configuration is used to balance a payload connected to a rotating link. All the parameters within the design vector $v$ are illustrated. The angles that need to be known for the kinematic relation are also shown.

The coordinates of point $C\left(x_{c}, y_{c}\right)$ can be written as:

$$
\begin{aligned}
& x_{c}=L_{c} \cdot \cos (\theta) \\
& y_{c}=L_{c} \cdot \sin (\theta)
\end{aligned}
$$

The distance between point $A$ and $C\left(L_{a c}\right)$ and the angle of the line connecting these points $\left(\phi_{a c}\right)$ can now be calculated.

$$
\begin{aligned}
L_{a c} & =\sqrt{\left(x_{c}-x_{0}\right)^{2}+\left(y_{c}-y_{0}\right)^{2}} \\
\phi_{a c} & =\operatorname{atan}\left(\frac{y_{c}-y_{0}}{x_{c}-x_{0}}\right)
\end{aligned}
$$

With all the lengths within triangle $\mathrm{ABC}$ known, the cosine rule can be used to determine the angles between all the links. The length of the links from point $\mathrm{A}$ to $\mathrm{B}$ and $\mathrm{B}$ to $\mathrm{C}$ are denoted as $L_{a}$ and $L_{b}$. The inner angles $\alpha$ and $\gamma$ can be found by the following equations.

$$
\begin{aligned}
& \alpha=\operatorname{acos}\left(\frac{L_{a}^{2}+L_{a c}^{2}-L_{b}^{2}}{2 \cdot L_{a} \cdot L_{a c}}\right) \\
& \gamma=\operatorname{acos}\left(\frac{L_{b}^{2}+L_{a c}^{2}-L_{a}^{2}}{2 \cdot L_{b} \cdot L_{a c}}\right)
\end{aligned}
$$

The angles $\alpha$ and $\gamma$ can be used to find the orientation of the links of the balancing component $\left(L_{a}\right.$ and $\left.L_{b}\right)$. The angle at point $A$ is $\phi_{a}$ and the angle at point $B$ is $\phi_{b}$.

$$
\begin{aligned}
& \phi_{a}=\phi_{a c}+\alpha \\
& \phi_{b}=-(\alpha+\gamma)
\end{aligned}
$$

The last unknown angle is at point $C\left(\phi_{c}\right)$, where the balancing component is connected to the unbalanced function. The cosine rule is again used to determine the angle. $\phi_{c}$ can be found by defining all the lengths within triangle BCD. When the distance between point $B$ and $D\left(L_{b d}\right)$ is calculated, $\phi_{c}$ can be determined. The length of the link from point $\mathrm{D}$ to $\mathrm{C}$ is denoted as $L_{c}$.

$$
\begin{aligned}
L_{b d} & =\sqrt{\left(x_{0}+L_{a} \cdot \cos \left(\phi_{a}\right)\right)^{2}+\left(y_{0}+L_{a} \cdot \sin \left(\phi_{a}\right)\right)^{2}} \\
\phi_{c} & =\operatorname{acos}\left(\frac{L_{b}^{2}+L_{c}^{2}-L_{b d}^{2}}{2 \cdot L_{b} \cdot L_{c}}\right)
\end{aligned}
$$

The balancing component may have a counterclockwise configuration, see Fig. 3. The angles $\phi_{a}$ and $\phi_{b}$ are defined differently in this case.

$$
\begin{aligned}
& \phi_{a}=\phi_{a c}-\alpha \\
& \phi_{b}=\alpha+\gamma
\end{aligned}
$$

\subsection{Energy approach}

When no friction is present in a mechanism and has a constant potential energy, the only required effort to move the system comes from the acceleration of that system [1]. The focus is thus to have a constant potential energy. The total potential energy $U_{\text {total }}$ of the system consists of gravitational energy and elastic potential. The gravitational energy comes from the payload and the weight of the links. The elastic potential comes from the torsional stiffness of the joints. The total potential energy $U$ can be written as:

$$
U_{\text {total }}=\sum_{1}^{n} \frac{1}{2} \cdot k_{i} \cdot\left(\phi_{i}-\phi_{i 0}\right)^{2}+\sum_{1}^{w} m_{j} \cdot g \cdot h_{j}
$$

The number of joints with a torsional stiffness is denoted by $n$. Every $i^{t h}$ joint has a stiffness $k_{i}$, an angular rotation $\phi_{i}$ and an initial angle $\phi_{i 0}$. The number of bodies with a weight are denoted by $w$ and $g$ as the gravitational acceleration. The $j^{t h}$ weight has a weight of $m_{j}$ and the height of that weight is $h_{j}$. The height is taken relative to $y_{m 0} \cdot y_{m 0}$ is the lowest point of the payload along its range of motion. This is used as reference point to determine 
the potential energy of the weights. ple is:

The potential energy of the spring at point $A\left(U_{S A}\right)$ for exam-

$$
U_{s A}=\frac{1}{2} \cdot k_{a} \cdot\left(\phi_{a}-\phi_{a 0}\right)^{2}
$$

Figure 4 indicates that all three links have a weight. The gravitational energy of the links and the payload $U_{m}$ are calculated with the following equations. The mass of the payload is taken as $m$. The payload rotates around point $D(0,0)$ with a radius of $r$ and with $\theta$ as degree of freedom. The gravitational energies of the links from point $A$ to $B, B$ to $C$ and $D$ to $E$ are denoted as $U_{m a}$, $U_{m b}$ and $U_{m c}$.

$$
\begin{aligned}
U_{m a} & =m_{a} \cdot g \cdot\left(\frac{1}{2} \cdot L_{a} \cdot \sin \left(\phi_{a}\right)+y_{0}-y_{m 0}\right) \\
U_{m b} & =m_{b} \cdot g \cdot\left(\frac{1}{2} \cdot\left(y_{0}+L_{a} \cdot \sin \left(\phi_{a}\right)+y_{c}\right)-y_{m 0}\right) \\
U_{m c} & =m_{c} \cdot g \cdot\left(\frac{1}{2} \cdot r \cdot \sin (\theta)-y_{m 0}\right) \\
U_{m} & =m \cdot g \cdot r \cdot \sin (\theta)
\end{aligned}
$$

The total energy function that needs to be optimized can now be written as:

$$
U_{\text {total }}=U_{s A}+U_{s B}+U_{s C}+U_{s D}+U_{m a}+U_{m b}+U_{m c}+U_{m}
$$

\subsection{Optimization}

The total energy curve is dependent on all the parameters within the design vector $\mathbf{v}$. This gives a large number of possible solutions. Furthermore, the effect of a single parameter on the total energy curve is an arduous task to determine analytically. Countless of possible solutions can be found by changing the relation between parameters. For example: a change in stiffness may still give a desired energy curve if the initial angle of that spring or the location of attachment point $A$ is changed.

A genetic algorithm (GA) was used to find sets of parameters within design vector $\mathbf{v}$ that give a desired outcome. A genetic algorithm is a search technique to find global optima of complex search problems [15]. The GA allows a large number of solutions to be tested and returns the solution with properties closest to the given objective function. It is a possibility that the algorithm converges towards local optima. As a result a returned solution by the genetic algorithm is not necessarily the best possible solution. However, when the number of tested solutions is large enough and is uniformly distributed along the search space, the global optimum is likely to be found.

The objective function is to minimize the fluctuation of the total energy curve over the range of motion $\left(\frac{\delta U_{\text {total }}}{\delta s}\right)$, Eqn. 22 .
When the fluctuation of the energy curve is zero, the outcome is a constant potential energy. To minimize stresses within the joints, the total energy level is also minimized. The mean of the total energy curve $\bar{U}(\mathbf{v})$ is used as measure for the energy level, Eqn. 23.

$$
\begin{aligned}
K & =\int_{a}^{b}\left(\frac{\delta U_{\text {total }}}{\delta s}(\mathbf{v}, s)\right)^{2} d s \\
\bar{U}(\mathbf{v}) & =\frac{1}{b-a} \int_{a}^{b} U_{\text {total }}(\mathbf{v}, s) d s
\end{aligned}
$$

$U_{\text {total }}(\mathbf{v}, s)$ is the potential energy of the system as function of the design vector $\mathbf{v}$ at location $\mathbf{s}$. The range of motion of the payload is from $a$ to $b$. $K$ denotes the value for the energy fluctuation. The change in potential energy $\left(\frac{\delta U \text { total }}{\delta s}\right)$ is squared to lower the peaks.

A weight factor $\lambda$ is introduced to indicate the priority of one of the optimizations. If $\lambda>1$, a higher priority is given to a constant potential energy level. This paper will consider a constant potential energy as primary objective: $\lambda=10$. The objective function can be written as:

$$
\min _{\mathbf{v}}(\bar{U}(\mathbf{v})+K \cdot \lambda)
$$

\subsection{Evaluation of optimization}

As the energy level is considered a secondary objective and does not influence the functionality of the system, it is not taken into consideration for the evaluation.

The system is optimized over a certain range of motion. Fluctuations in the energy curve indicate the need for an external force or moment to keep the system in equilibrium. For the evaluation, the maximum required force or moment is compared between the unbalanced and balanced function.

An unbalanced function can describe a rotation or translation. The corresponding maximum moment $M$ or force $F$ of an optimized system will be considered. The maximum moment $M_{u}$ or force $F_{u}$ of the unbalanced function are used as reference. The percentage of moment and force reduction is calculated as followed:

$$
\begin{aligned}
\operatorname{Reduction}(M) & =100-\left(100 / \max \left(\left|M_{u}\right|\right)\right) \cdot \max (|M|) \% \\
\operatorname{Reduction}(F) & =100-\left(100 / \max \left(\left|F_{u}\right|\right)\right) \cdot \max (|F|) \%
\end{aligned}
$$

The resulting force and moment can be calculated by taking the derivative of the total potential energy function. Taking the derivative with respect to the displacement $y$ gives the resulting 
force in that direction, Eqn. 277. Taking the derivative with respect to the rotation $\theta$ gives the resulting moment, Eqn. (28) [16].

$$
\begin{gathered}
-\frac{\delta U_{\text {total }}}{\delta y}=F \\
-\frac{\delta U_{\text {total }}}{\delta \theta}=M
\end{gathered}
$$

\section{EXAMPLES}

The designed method allows numerous possibilities. This section presents three examples. The first two show a mass connected to a rotating link with two possible configurations of balancing components. The third example shows the mass as vertically translating surface. The links of the balancing component are chosen to have equal length. The weight of the links were assumed to be $0.3024[\mathrm{~kg} / \mathrm{m}]$. The assumption for this weight is based on the use of aluminum u-profiles with sides of $20[\mathrm{~mm}]$ and a wall thickness of $2[\mathrm{~mm}]$.

In these examples, the effect of friction, damping and other non-conservative forces are neglected. The values used for the parameters are listed in Tab. 1 and the parameters are shown in Fig. 4 All unbalanced functions in the examples have a payload of $1[\mathrm{~kg}]$ and are moved over a height of $0.6[\mathrm{~m}]$.

\subsection{Single clockwise balancing component}

This first example shows the payload connected to the end of a rotating link, see Fig. 5a. The balancing component is connected to the unbalanced function with a clockwise configuration. The system is optimized to be balanced from $\theta \in\left[-\frac{\pi}{2}: \frac{\pi}{2}\right]$ [rad], where $\theta$ is denoted as the degree of freedom. Figure $5 \mathrm{~b}$ shows the potential energies within the system. The moment required to keep the system in static equilibrium along its range of motion is plotted in Fig. 5c Table 1 shows the values for parameters used in this example (1). This example has a peak moment reduction of $85,95 \%$.

\subsection{Double balancing component}

This second example has two balancing components connected to the unbalanced function, Fig. 6a. The unbalanced function consists out of a payload attached to the endpoint of a rotating link. The system is optimized to be balanced from $\theta \in\left[-\frac{\pi}{2}: \frac{\pi}{2}\right][\mathrm{rad}]$. Figure $6 \mathrm{~b}$ shows the potential energies within the system. The moment required to keep the system in static equilibrium along its range of motion is plotted in Fig. 6c. Table 1 shows the values for parameters used in this example (2). This example has a peak moment reduction of $94,09 \%$.

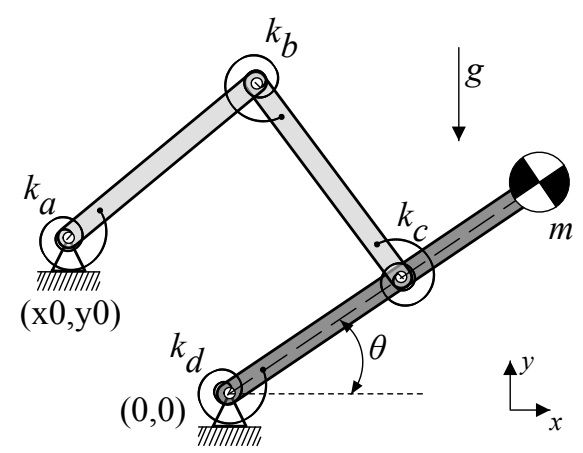

(a)

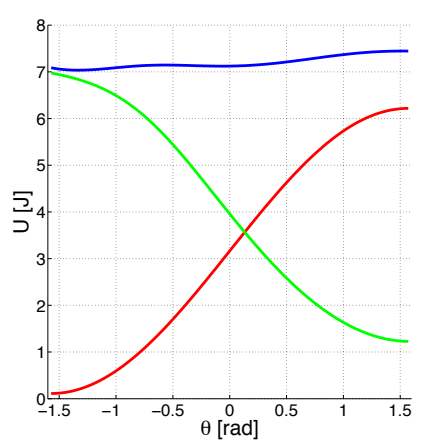

(b)

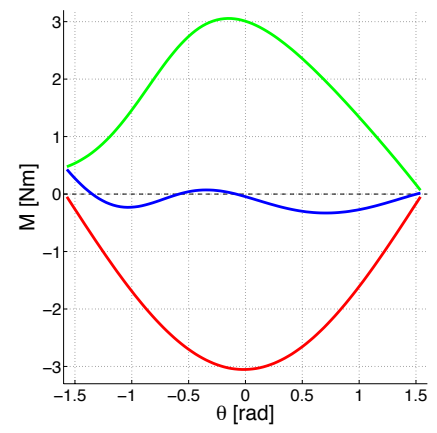

(c)
FIGURE 5: (a) An example of a single clockwise balancing component attached to a payload connected to a rotating link. The energy curve of the system is shown in $(b)$. The red line represents the unbalanced function $\left(U_{m c}+U_{m}\right)$, the green line represents the balancing component $\left(U_{s A}+U_{s B}+U_{s C}+U_{s D}+U_{m a}+\right.$ $\left.U_{m b}\right)$ and the blue line represents the curve of the total system $\left(U_{\text {total }}\right)$. The corresponding moments are plotted in $(c)$.

\section{3 (removed due to confidentiality) 4 CONSTRUCTION AND EVALUATION}

This section presents the construction and evaluation of a gravity balancer with compliant joints. The demonstrator is evaluated to validate the presented method. The demonstrator will have a similar orientation as presented in the first example: a single balancing component in clockwise configuration connected to a rotating link. The range of the demonstrator has been chosen, such that the selected springs stay within certain limits of their maximum rotation [17]. The demonstrator is optimized from $-\pi / 3$ to $\pi / 2$ [rad]. To simplify fabrication, springs with similar size and stiffness have been chosen.

\subsection{Fabrication}

Before physical construction, the type of compliant joints and weight of the construction need to be known. This is step five (5) within the presented design method. Helical torsional 


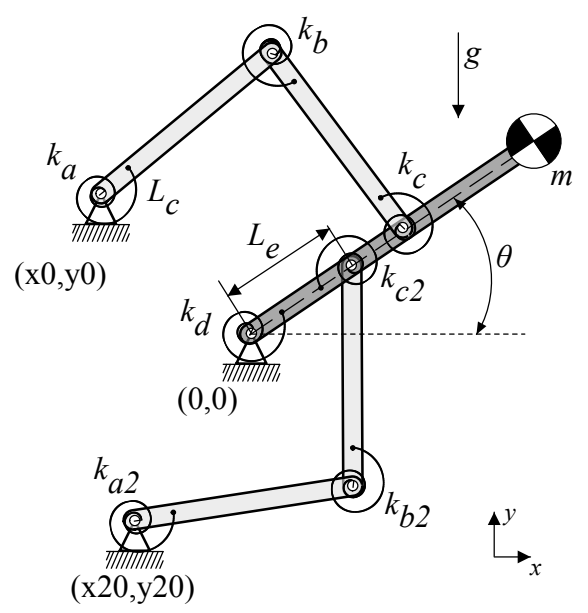

(a)

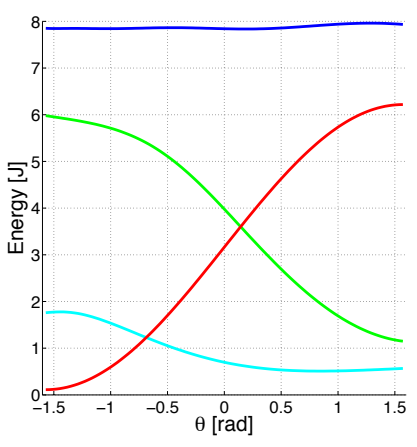

(b)

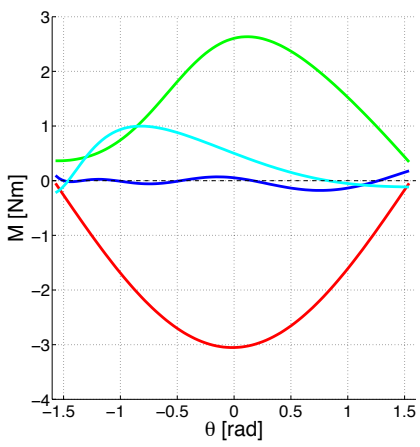

(c)
FIGURE 6: (a) An example of two balancing components attached to a payload connected to a rotating link. The two balancing components have a clockwise and counterclockwise configuration. The energy curve of the system is shown in (b). The red line represents the unbalanced function $\left(U_{m c}+U_{m}\right)$, the green line represents the clockwise balancing component $\left(U_{s A}+U_{s B}+U_{s C}+U_{s D}+U_{m a}+U_{m b}\right)$, the light blue line represents the counterclockwise balancing component $\left(U_{S A 2}+U_{S B 2}+\right.$ $\left.U_{s C 2}+U_{m a 2}+U_{m b 2}\right)$ and the dark blue line represents the curve of the total system $\left(U_{\text {total }}\right)$. The corresponding moments are plotted in $(c)$.

springs were chosen as compliant joints as they can be ordered 'off-the-shelf'. To reduce friction from the coils being pressed together, springs were ordered from which the coils do not touch. When two links are connected with a helical torsion spring, no rigid joints are required, see Fig. 7 p.

Together with the actual weight of the links, the total weight of the construction can be measured (step 5). The next step was to again optimize the parameters (step 3). After another evaluation of the results (step 4), it was seen that the components were
TABLE 1: The values of parameters and evaluation of examples. The parameters are shown in Fig. 4

\begin{tabular}{|c|c|c|c|}
\hline Example & 1 & 2 & Unit \\
\hline$x_{0}$ & -0.216 & -0.256 & {$[\mathrm{~m}]$} \\
\hline$y_{0}$ & 0.195 & 0.059 & {$[\mathrm{~m}]$} \\
\hline$x_{20}$ & - & 0 & {$[\mathrm{~m}]$} \\
\hline$y_{20}$ & - & -0.162 & {$[\mathrm{~m}]$} \\
\hline$k_{a}$ & 0.984 & 0.955 & {$\left[\frac{\mathrm{Nm}}{\mathrm{rad}}\right]$} \\
\hline$k_{b}$ & 0.989 & 0.312 & {$\left[\frac{\mathrm{Nm}}{\mathrm{rad}}\right]$} \\
\hline$k_{c}$ & 0.999 & 0.996 & {$\left[\frac{\mathrm{Nm}}{\mathrm{rad}}\right]$} \\
\hline$k_{a 2}$ & 0.157 & 0.281 & {$\left[\frac{\mathrm{Nm}}{\mathrm{rad}}\right]$} \\
\hline$k_{b 2}$ & - & 0.215 & {$\left[\frac{\mathrm{Nm}}{\mathrm{rad}}\right]$} \\
\hline$k_{c 2}$ & - & 0.406 & {$\left[\frac{\mathrm{Nm}}{\mathrm{rad}}\right]$} \\
\hline$k_{g}$ & - & 0.105 & {$\left[\frac{\mathrm{Nm}}{\mathrm{rad}}\right]$} \\
\hline$\phi_{a 0}$ & 1.871 & 1.884 & $0[\mathrm{rad}]$ \\
\hline$\phi_{b 0}$ & -2.293 & -2.227 & $0[\mathrm{rad}]$ \\
\hline$\phi_{c 0}$ & 2.581 & 1.888 & [rad] \\
\hline$\phi_{d 0}$ & 0.993 & 0.973 & {$[\mathrm{rad}]$} \\
\hline$\phi_{a 20}$ & - & -0.512 & [rad] \\
\hline$\phi_{b 20}$ & - & 0.660 & [rad] \\
\hline$\phi_{c 20}$ & - & 0.344 & {$[\mathrm{rad}]$} \\
\hline$L_{a}$ & 0.3 & 0.3 & {$[\mathrm{~m}]$} \\
\hline$L_{b}$ & 0.3 & 0.3 & {$[\mathrm{~m}]$} \\
\hline$L_{c}$ & 0.25 & 0.25 & {$[\mathrm{~m}]$} \\
\hline$L_{e}$ & - & 0.25 & {$[\mathrm{~m}]$} \\
\hline$r$ & 0.3 & 0.3 & {$[\mathrm{~m}]$} \\
\hline$a$ (start) & $-\pi / 2[\mathrm{rad}]$ & $-\pi / 2[\mathrm{rad}]$ & \\
\hline$b$ (end) & $\pi / 2[\mathrm{rad}]$ & $\pi / 2[\mathrm{rad}]$ & \\
\hline Reduction & $85,95 \%$ & $94,09 \%$ & \\
\hline
\end{tabular}

able to give a desired outcome. The last step was to build the construction (step 6). The complete assembled demonstrator is shown in Fig. 7. The optimized parameters used for the construction of the demonstrator are shown in Tab. 2 . 

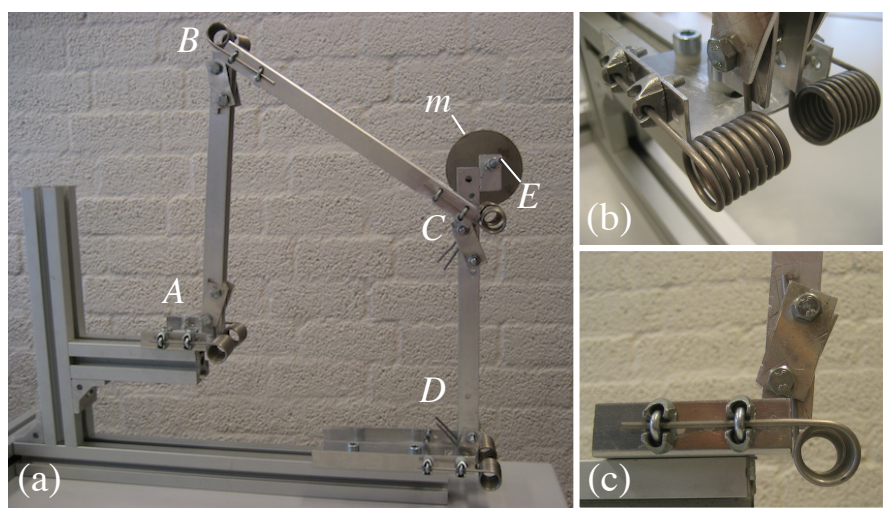

FIGURE 7: The completely assembled demonstrator is shown in (a). A detailed view of the connection between two links is shown in (b), where can be seen that no rigid joints are used. A detailed view of how the links are connected to the torsion spring is shown in (c).

TABLE 2: Parameters of the demonstrator

\begin{tabular}{lll}
\hline Demonstrator & values & Unit \\
\hline$m$ & 1 & {$[\mathrm{~kg}]$} \\
$x_{0}$ & -0.27 & {$[\mathrm{~m}]$} \\
$y_{0}$ & 0.12 & {$[\mathrm{~m}]$} \\
$k_{a}$ & 1.069 & {$\left[\frac{N m}{r a d}\right]$} \\
$k_{b}$ & 1.035 & {$\left[\frac{N m}{r a d}\right]$} \\
$k_{c}$ & 1.035 & {$\left[\frac{N m}{r a d}\right]$} \\
$k_{d}$ & 0.990 & {$\left[\frac{N m}{r a d}\right]$} \\
$\phi_{a 0}$ & 2 & {$[\mathrm{rad}]$} \\
$\phi_{b 0}$ & -2.3 & {$[\mathrm{rad}]$} \\
$\phi_{c 0}$ & 2.405 & {$[\mathrm{rad}]$} \\
$\phi_{d 0}$ & 0.905 & {$[\mathrm{rad}]$} \\
$L_{a}$ & 0.3 & {$[\mathrm{~m}]$} \\
$L_{b}$ & 0.3 & {$[\mathrm{~m}]$} \\
$L_{c}$ & 0.25 & {$[\mathrm{~m}]$} \\
$r$ & 0.3 & {$[\mathrm{~m}]$} \\
\hline
\end{tabular}

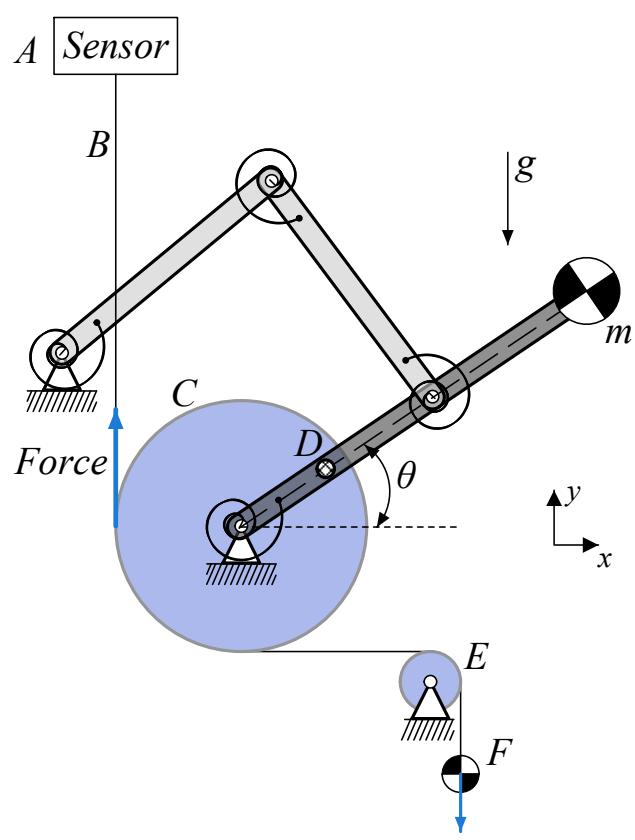

FIGURE 8: The experimental setup of the demonstrator. A vertical tensile strength tester $(A)$ is used for the measurement of the force in the cable $(B)$. The cable moves along a disc $(C)$ with constant radius. The disc is used to enable the conversion of the measured force to a moment. The demonstrator is connected to the disc with a shaft (D). To ensure tension in the cable, a weight (F) has been added. The extra weight is connected to the disc with a pulley $(E)$.

\subsection{Experimental evaluation}

An experimental setup has been constructed to evaluate the performance of the demonstrator, Fig 8 . Along the systems range of motion, the moment required to keep the system in equilibrium is measured. A vertical tensile strength tester is used for the measurement of the force. A disc with constant radius is used to enable the conversion of the measured force to a moment. The demonstrator is connected to the disc with a shaft. A weight has been added to ensure tension in the cable.

To determine the energy loss, the measurements are performed in two directions to obtain a hysteresis loop. The energy loss is assumed to be equal in both directions. The resulting average line in between the hysteresis loop is then an approximation of the required force if there would be no energy loss. During the measurement, the demonstrator is moved with low and constant speed $(0.003 \mathrm{~m} / \mathrm{s})$ to be able to neglect inertia effects. 


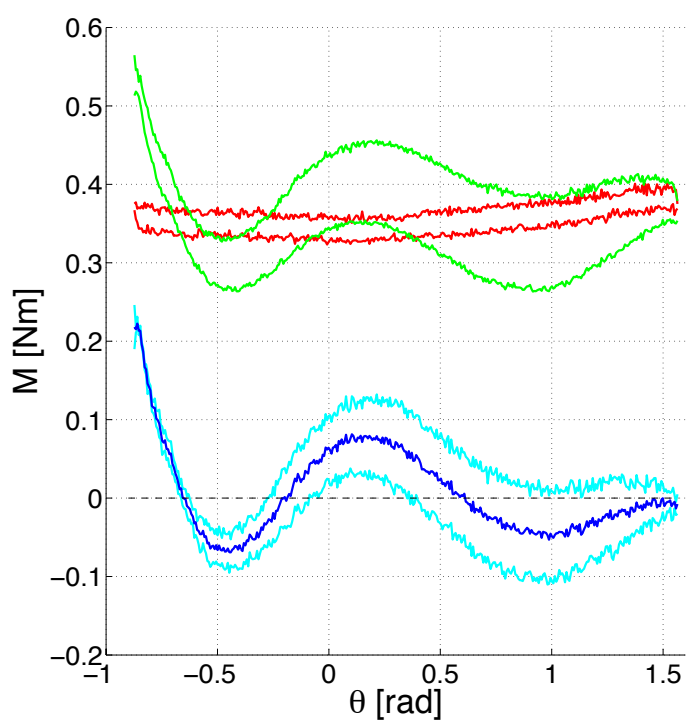

FIGURE 9: The measurements of the demonstrator. The red curve shows the measurement of the experimental setup without the demonstrator. The green curve shows the measurements of the demonstrator with entire setup. The light blue curve shows the difference between green and red: the hysteresis curve of the demonstrator. The dark blue curve indicates the required moment to keep the demonstrator in equilibrium if no hysteresis is present.

TABLE 3: Summary of results.

\begin{tabular}{lll}
\hline & Unit & Result \\
\hline Theoretical reduction & $\%$ & 95,6 \\
Measured reduction & $\%$ & 92,98 \\
\hline
\end{tabular}

\subsection{Measurement results}

To check the internal error of the test setup, first the constant weight of a mass was measured. The fluctuation around the expected value would imply an error within the experimental setup. From the hysteresis loop of this measurement, the error within the test setup could be determined.

The demonstrator was measured together with the additional weight. By taking the difference between the measurement of the demonstrator and the measurement of the weight, the actual required moment could be determined. The measurement results of the mass and the demonstrator are shown in Fig. 9 The theoretical values are compared with the actual measured values in Fig. 10. Table 3 shows a summary of the results.

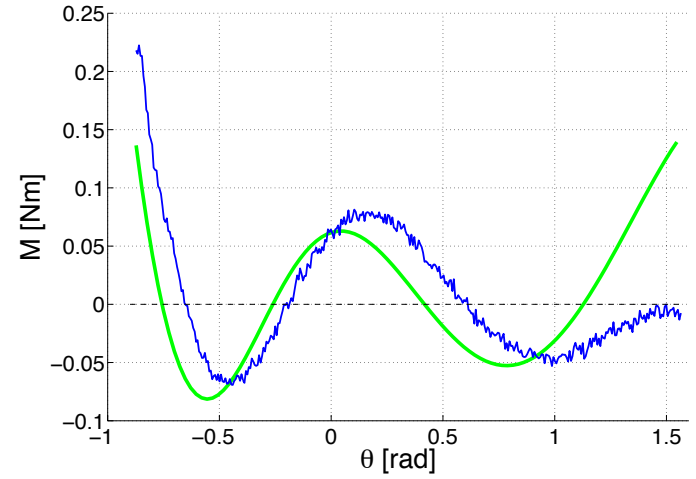

FIGURE 10: Comparison of theoretical and measured curves. The green curve shows the theoretical value and the blue curve shows the measured values.

\section{DISCUSSION}

This section will evaluate the applied method. The results will be discussed followed by an evaluation of the demonstrator. Finally recommendations are given for further research and development.

\subsection{Evaluation of applied method}

The applied method is based on obtaining a constant potential energy. An alternative would be to analyze the forces. Energy is a scalar quantity whereas forces are vector quantities. Consequently, the use of potential energy allows a simplified analysis with respect to the use of forces. Another advantage is the ability to manipulate the energy level to minimize stresses within the joints. This is important when considering the safety of the system.

The developed method can be said to be general. The results show an example of a rotating as well as a translating mechanism. Regardless of the type of motion, this method allows any number of balancing components to be attached to the unbalanced function which has a single degree of freedom. The unbalanced function can be an existing mechanism or one that has been newly created.

The use of GA to find values for parameters reduces the insight of the designer into the effect of individual parameters on the total system. The configuration and number of balancing components should be tested before knowing the effect. It is a trial and error process. There is no guarantee that a constant potential energy curve will be produced. However, a combination of parameters that lead to the approximation of a constant potential energy is likely to be found if it exists.

The addition of a second balancing component has shown to lead to a better approximation of static balance (Figs. 5, 6 Tab. 11. The added energy storage elements however, do increase the total energy level. Even though the total energy level 
increases, it can be seen that the required energy level of the balancing components are lower. This is caused by the fact that the required storage of potential energy is now divided amongst more springs. The lower energy level indicates less energy stored within the springs, which indicates lower stresses. The system is hereby made safer and the balancing of a larger weight could be made possible. Lower stresses is of special use for compliant joints, as stresses due to large angular rotations are a limiting factor.

For the evaluation of the examples, the unbalanced function is taken as reference. For the first two examples, the payload is connected to a rotating link. The spring $\left(k_{d}\right)$ connecting this link to the base is considered part of the balancing component. The stiffness $k_{d}$ and initial angle $\phi_{d 0}$ are chosen to be part of the design vector. It should be considered that the outcome of the evaluation depends on how the unbalanced function is defined.

The applied method shows the potential for many novel solutions for gravity balancers using compliant joints or any other form of torsional stiffness. The method takes a torsional stiffness at every pivot point into account, so it is possible that created mechanisms gain all their motion from the deflection of material. This indicates the potential of a fully compliant gravity balancer with monolithic structure. This is considered to be a valuable contribution in the field of static balancing of compliant mechanisms (SBCM).

No solution has been found which has exact static balance. The solutions all have fluctuating energy curves which indicates that the objective function is not met completely. However, adding balancing components has shown to give the possibility for a better approximation of constant potential energy, Tab. 1 Because the energy functions have many variables they are difficult to analyze mathematically. The applied optimization method of genetic algorithms finds solutions numerically, but there is no guarantee that such an algorithm converges to the global optimum. Examining the energy functions analytically is complex, but could give more insight into the behavior of the system as function of the separate variables. Also looking at the objective function analytically could give better solutions or a better idea if exact static balance would be possible.

\subsection{Evaluation of demonstrator}

The use of helical torsion springs was chosen. The advantages of these springs are low costs, 'off-the-shelf' availability and a large range of motion. A disadvantage is the 'axis-drift' that these springs exhibit due to forces in the vertical plane. As the spring is stretched in the vertical plane the axis of rotation 'drifts' away. The springs also have a low stiffness perpendicular to the motion plane (off-axis stiffness). However, it is assumed that no out of plane forces are introduced to the system.

The demonstrator has successfully validated the presented method. The difference between the measured values and theo- retical values are considered to be little, Fig. 10. The difference can be explained as follows.

In the method, the energy stored in the springs is solely based on the rotation of the springs. As the springs are also stretched when the payload moves downward, additional energy is stored. The axis drift was measured in the springs at point A, where the springs are stretched up to $\sim 4[\mathrm{~mm}]$. The springs were also measured separately on tension. The axis drift caused by stretch in point $\mathrm{A}$ adds $\sim 0.026[J]$ to the system. With the assumption that the springs at the other pivot points store a similar amount of energy, it can be said there is an additional energy of $\sim 0.1[J]$. The additional energy stored in the springs causes the balancing component to apply a larger moment. The larger moment results in an upward shift of the total moment curve, which can be seen in the left two peaks in Fig. 10

During assembly, an error can be introduced within the initial angles of the springs and within the attachment of endpoints of the links to the base. These errors are likely to introduce the horizontal shift to the right and the error within the right peak.

From Fig. 9 can be seen that the demonstrator shows hysteresis. The hysteresis within the demonstrator is mainly caused by friction within the springs and between the shaft and the link, Fig. 8. Axis drift at point A causes the connected link to rotate about a different axis than the shaft. As a result, the shaft slides along the connected link and additional friction is introduced. As the springs rotate, the coils come into contact with each other and cause friction. The use of different compliant joints, where no frictional contact is present would reduce hysteresis considerably.

\subsection{Innovation}

This paper presented a number of innovations. The innovations are listed below:

A new method has been developed. This method gives the possibility to create gravity balancers with different types of compliant joints. These may be used to accommodate numerous applications. The gravity balancers consist of rigid links connected with compliant joints.

The method created a way to use existing compliant joints for a new application. As the deformation of the material is used as source of motion, rigid joints with bearings may now be replaced with compliant joints. The deformation of material is also used for energy storage. As existing compliant joints can be used, the created mechanisms do not require any complex or novel fabrication. The current field of application of existing compliant joints is hereby extended considerably.

For the first time a gravity balancer has been constructed where all the rigid joints are replaced with compliant joints. 
Consequently, it is now possible to design gravity balancers which gain all their motion from the deflection of material. This can lower friction, reduce backlash and reduce the number of parts within the mechanism.

\subsection{Recommendations}

Gravity balancers are often intended to balance various weights. It is suggested that the mechanism is made adjustable to balance various weights to have a larger field of application. The adjustment could for example be realized by changing the relative distance between attachment points of the links. Another possible way to adjust the system to balance a different weight would be to make the stiffness or initial angle of a joint adjustable.

Compliant joints have the disadvantage that they may exhibit axis drift and low off-axis stiffness. The resistance against axis drift and out of plane forces could be increased by using a different type of compliant joint. The presented method uses rigid body kinematics, which assumes zero axis-drift. Different types of compliant joints have their own unique characteristic. The method could predict the behavior of the system better if the effect of axis-drift would be implemented.

Now off-the-shelf helical torsion springs are used. Using compliant joints as developed by Trease [8] with little axis drift and high off-axis stiffness could increase the performance considerably. When the compliant joints are custom made, the initial angles could be manufactured to specification. This would simplify assembly and reduce the error introduced during assembly.

The balancing component can be extended by additional links, thereby creating a chain of links with more compliant joints. The energy of the system would then be stored amongst more compliant joints which can lead to smaller stresses. This would be an advantage especially for monolithic structures, which are often limited by their yield strength. As a result, this could lead to a lot more possibilities in the design of monolithic compliant gravity balancers.

\section{CONCLUSIONS}

This paper had the goal to develop a method for the design of gravity balancers which use compliant joints in stead of conventional rigid body joints. The second goal was to construct a demonstrator. Multiple examples have been presented. By building and testing a demonstrator, the advantages of gravity balancing and compliant joints have successfully been combined. Both goals have been met. The conclusions can be listed as follows:

- A new method has been developed to create gravity balancers where all the rigid joints are replaced with compliant joints.
- By using the deformation of material as source of motion and source of energy storage, the current field of application of existing compliant joints is extended considerably.

- For the first time a gravity balancer has been constructed which gains all its motion from the deflection of material. The demonstrator has a peak moment reduction of $93 \%$.

- The presented method is extensible and bears great promise in the design of fully compliant monolithic gravity balancers.

\section{ACKNOWLEDGMENT}

This research has been done at InteSpring B.V. and is part of the "ElastiCom-project", KWR09084. The author wishes to thank the team at InteSpring B.V. for a stimulating work environment and constructive feedback.

\section{REFERENCES}

[1] Herder, J.L., Energy free systems: Theory, conception and design of statically balanced spring mechanisms, Ph.D. Thesis, Dep. of Mechanical Engineering, Delft University of Technology, The Netherlands, 2001.

[2] Herder, J.L. On Static Balancing in Parallel Mechanisms Proceedings of the WORKSHOP on Fundamental Issues and Future Research Directions for Parallel Mechanisms and Manipulators. Quebec City, Quebec, Canada, October, 2002.

[3] Gopalswamy, A., Gupta, P., and Vidyasagar, M.,. A new parallelogram linkage configuration for gravity compensation using torsional springs. In Robotics and Automation. Proceedings., 1992 IEEE International Conference on, pp. 664669 vol.1, 1992.

[4] Arakelian, V., Ghazaryan, S. Improvements of balancing accuracy of robotic system: Application to leg orthosis for rehabilitation devices. Mechanism and Machine Theory 43, 2008.

[5] Stienen, A., Edsko E., Hekman E., Van der Helm F., Prange G., Jannink M., Aalsma M., and Van der Kooi H. Freebal: dedicated gravity compensation for the upper extremities. article, 2007.

[6] InteSpring B.V., www.intespring.nl

[7] Howell, L. L. Compliant Mechanisms. John Wiley and Sons Inc., New York,US, 2001.

[8] Trease, B., Moon Y., Kota S. Design of large-displacement compliant joints, ASME,2005

[9] Morsch F. Design of a generic zero stiffness compliant joint. M.Sc. Thesis, Delft University of Technology, Delft, The Netherlands, October, 2009. 
[10] Trease, B. and Dede, E. Statically Balanced Compliant Four-Bar Mechanism. ASME Student Mechanism Design Competition, University of Michigan, 2004.

[11] Claus, M. R. Gravity balancing using configurations of torsion bars; with application to the HCI foldable container. M.Sc. Thesis, Delft University of Technology, Delft, The Netherlands, December, 2008.

[12] Radaelli, G., Gallego, J. A., Herder, J. L., An energy approach to static balancing of systems with torsion stiffness, Proceedings of the ASME 2010 International Design Engineering Technical Conferences \& Computers and Information in Engineering Conference. Montreal, Quebec, Canada, August 15-18, 2010.

[13] Meriam, J. L. and Kraige L.G. Engineering Mechanics, Statics. John Wiley and Sons Inc., New York,US, Third Edition, 1993.

[14] Weinstein, W.D., "Flexural Pivot Bearings", Machine Design, June 10, pp. 150-157, 1965.

[15] Goldberg, D. Genetic Algorithms in Search, Optimization and Machine Learning. Addison-Wesley publishing company Inc, US. 1953.

[16] Meriam, J. L. and Kraige L.G. Engineering Mechanics, Dynamics. John Wiley and Sons Inc., New York,US, Third Edition, 1993.

[17] Lesjöfors, Technical Spring Catalogue, Brommatryck \& Brolins AB, Stockholm, 2008. 